\title{
The Initial Gamma Ray Transient Studies
}

\section{Thomas L. Cline ${ }^{1}$}

NASA Goddard Space Flight Center, Emeritus

Greenbelt, Maryland, 20771 USA

E-mail: Thomas.L.Cline@nasa.gov

The early studies of astronomical gamma ray transients are reviewed, with emphasis on our involvements and influences, including the first gamma-ray burst detections and the creation of the Interplanetary GRB Network. I interpreted the 1979 March 5 event as unique, whereas this initial IPN success ironically complicated and deepened the mystery of GRBs, with other views that its location at N49 must be accidental, or that it suggested neutron-star GRB origins. Also outlined are our Shuttle "Gamma-Dome" that inspired the CGRO-BATSE, the Ge GRB spectrometer, and the Solar Polar Mission - reduced to the successful but no longer stereoscopic Ulysses. Other developments, e.g., the resolution of the superflare/GRB confusion, are outlined.

Gamma-Ray Bursts 2012 Conference Munich, Germany

May 7-11, 2012

1 Speaker 
I shall review here my recollections of the early history of cosmic gamma ray bursts, and how this puzzle took nearly 30 years to unravel. The possibility of detecting astrophysical gamma ray transients first interested me with Colgate's prediction (1) in the late 1960s of highenergy gamma-rays to be emitted at the onset of a supernova. Funding was obtained in 1971 to begin on a one-meter-diameter balloon-borne instrument to investigate this possibility. Independently, there was the incidental detection of solar-flare gamma rays with my positron detector in the mid-1960s, inspiring me to revise my IMP-6 electron detector to have a 0.1-1 $\mathrm{MeV}$ photon capability. That mission was launched in 1971, and over the next two years it registered several intense increases of photon counts, but not at the times of solar flares.

The irony was that I was then preparing to look for the gamma-ray transients Colgate had suggested, and found these events instead. Rather than rushing into print, I asked experimenters on NASA missions for their possible confirmation. But, no luck, since all their detectors were highly directional and were in orbits underneath the Earth's trapped radiation, thus subject to count-rate increases of all sorts. I would not publish the detection of these bursts, that could be dismissed by skeptics as detector malfunction, without some confirmation.

The Vela discovery of gamma ray bursts (2) was published in April 1973. R. Klebesadel's list of over a dozen events found since 1967 included all of our IMP events. From the geometric timing (Fig. 1.), we can infer that a bomb test of the sort guarded against would have an instant onset followed by a smooth decay, easily distinguished from the chaotic profile of a GRB. (This discovery made the popular press: one tabloid claimed that aliens were conducting wars in outer space, but it quotes Klebesadel and me as disagreeing with that idea.) The timing of the events across the Vela orbits, separated by up to 1 light-second, made a determination of crude source locations possible, with accuracy enough to exhibit the lack of any clear pattern.

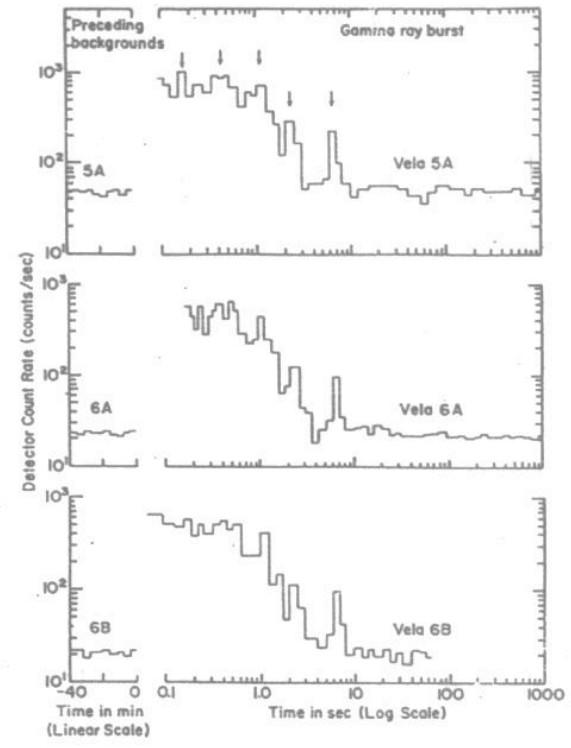

Fig. 1. The August 22, 1970 GRB.

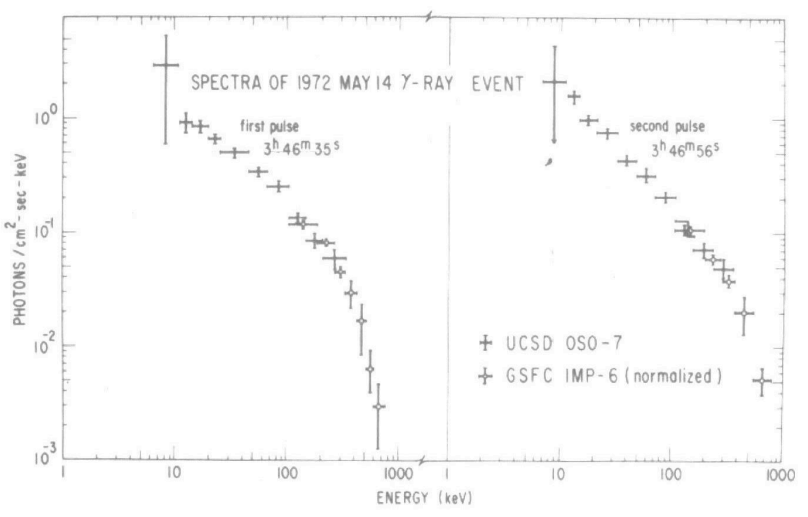

Fig. 2. The OSO/IMP May 14, 1972 GRB. 
With their publication, I suddenly became "the man who did not discover gamma ray bursts" but the one who accomplished the first confirmation of their discovery, with a talk at the summer-1973 Denver cosmic-ray conference (3) and a joint publication (4). Mel Ulmer then found one of our IMP events buried in the OSO data, which UCSD jointly published (5) as the second confirmation of the GRB discovery. The significance of this was that the event direction determined with a collimator agreed with that from the Vela timing technique. This source was at high galactic latitude, confirming the lack of any galactic disk pattern that might be expected.

The IMP results provided the first GRB spectral data: a $200-\mathrm{keV}$ signature that separated these events from known x-ray transient behavior. The IMP-6 spectrum fit onto that of the OSO event (fig. 2), and event similarity was repeated with dozens more IMP-6 and IMP-7 events. I pointed out in those early days that a typical GRB energy, if extrapolated to a cosmological distance, roughly equals one solar rest mass - too interesting a coincidence to ignore! In 1974, our supernova-gamma-ray search was balloon borne, and in May, 1975, we conducted perhaps the first simultaneous balloon flights in cosmic-ray history (6): B. Teegarden in South Dakota and myself in Texas. With 20 hours of overlap, this search for GRB events was also fruitless.

Soon after the GRB discovery, I asked NASA Headquarters to permit and support the modification of instrumentation under construction on interplanetary missions that would make possible accurate timing over great distances. HQ designated ISEE-1 and -3 and Helios-2 as possibilities for our 'piggy-back' detectors, and later awarded Los Alamos the opportunity on Pioneer Venus Orbiter. We were able to design and build a 1-kg piggy-back GRB detector for delivery in 1975 for the Helios-2, a German spacecraft. It was successfully launched in January 1976 with our instrument, the first ever flown purposefully for gamma-ray burst study.

A full IPN was completed two years later when PVO and ISEE-3 were launched. In addition, Veneras 11 and 12 also had GRB instrumentation from the Franco-Soviet collaboration formed by G. Vedrenne of Toulouse, who had been prompted by our talk at Denver to create instruments for Estulin's group in Moscow. This IPN, with PVO and the two Veneras near Venus, ISEE-3 and the Velas near the Earth, and Helios-2 in solar orbit, forming a triangle of interplanetary baselines, accomplished a number of GRB source locations with arcminute accuracy from 1978 through the early 1980s. Optical and radio-astronomy searches took many months to obtain scheduling, but always came up with empty source fields (e.g., Fig. 3), at first.

At that time, I had given our ISEE-3 piggy-back opportunity to B. Teegarden, who brilliantly devised a high-energy-resolution Germanium spectrometer, using the advantage of the ISEE-3 ecliptic-plane spin to provide passive, radiative cooling to cryogenic temperatures. This device operated satisfactorily, but provided no narrow-line GRB spectral features. Teegarden later devised a similar but improved radiatively-cooled spectrometer for flight on the GGS-Wind spacecraft, but that also yielded no spectroscopic breakthrough.

What changed everything was the detection by about a dozen missions of an extremely unusual transient on March 5, 1979. In our first paper (7), I interpreted this event as a 'unique' transient, with an IPN source fit (Fig 4.) onto the supernova remnant N49 in the LMC (8). My view (not endorsed by all the co-authors) was based on the anomaly of the onset (Fig 5.) of several orders of magnitude intensity rise in under 2 milliseconds, the pulsation (Fig. 6) noted by the Leningrad group (9), and this source fit to a known object, N49. All the data were 
consistent with a neutron-star origin and none were shared by any other gamma ray event. The reanalysis of the source position (10) was the most precise in gamma-ray astronomy (Fig. 7), $\sim 30$ arc-seconds from the center of N49. The Leningrad group on the Veneras had found two soft repeaters, one with a source direction compatible with that of the March 5 event (11). This was another property not shared by any GRB. I later republished my interpretation (12).

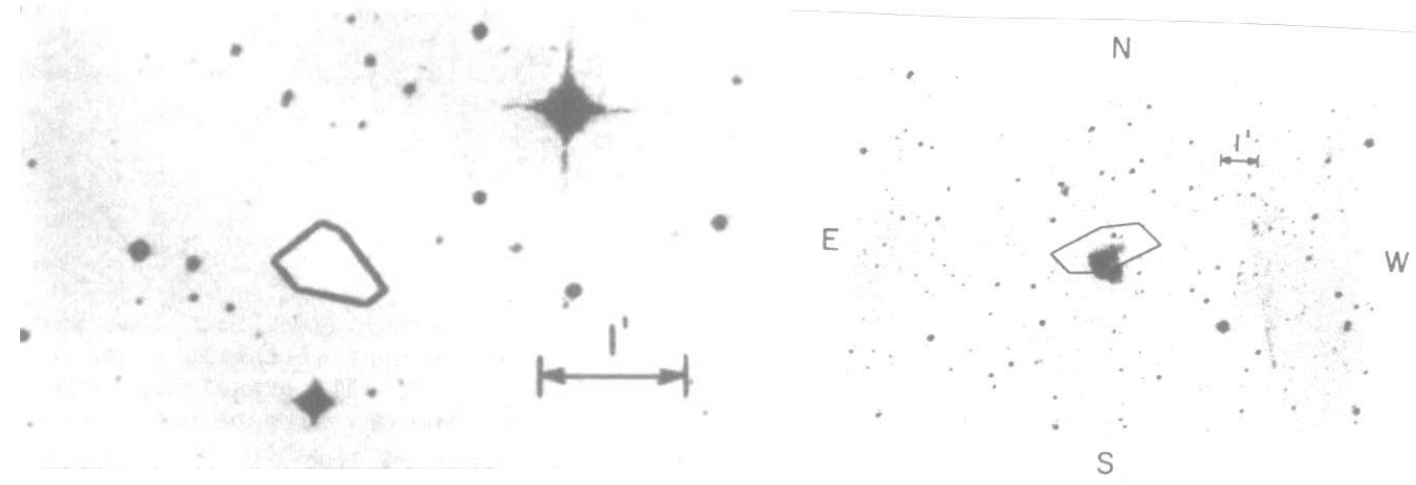

Fig. 3. A typical IPN empty source box.

Fig. 4. One source fit: N49 in the LMC.

It is ironic that this one IPN success actually deepened and confused the GRB mystery, given the previous claim of many astronomers that GRBs could not come from sources farther away than a few kpc, assuming that this event is a GRB. Yet many interpreted it as just an extreme GRB variation, and evidence that GRBs are from neutron stars. Every possible point of view was researched, as that should be, but the picture was plagued by contradictory premises. Complicating things further was early evidence for a bimodal GRB duration distribution (13).
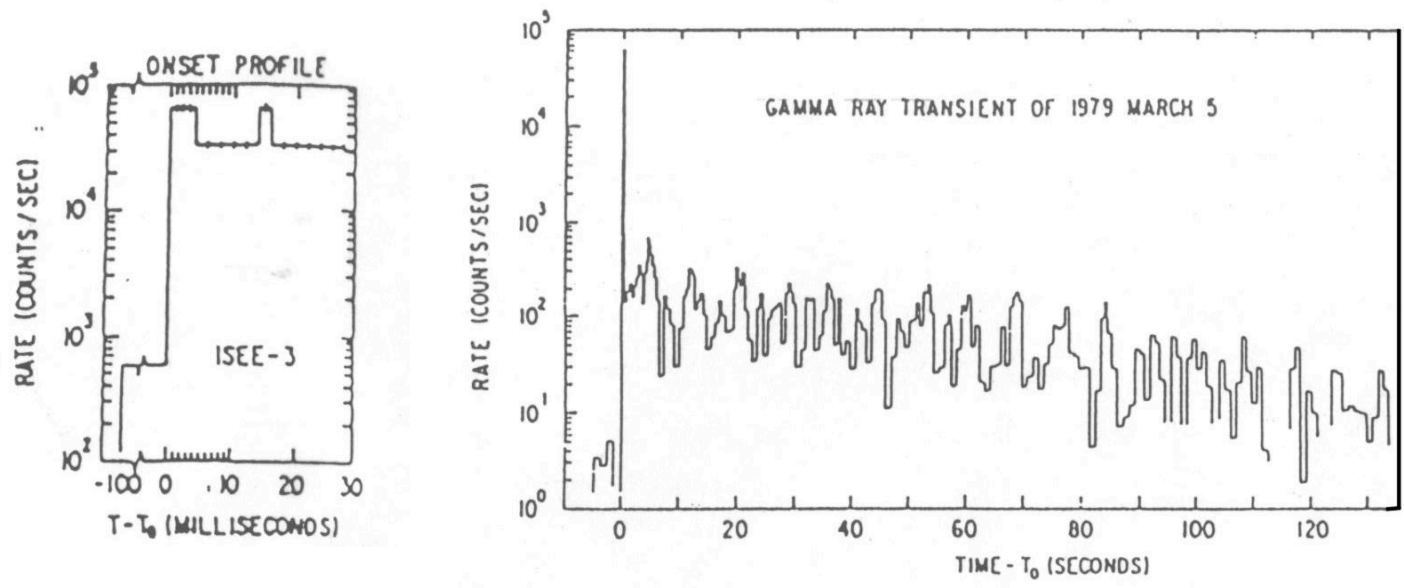

Fig. 5. The 2-ms risetime and, Fig. 6., the pulsing decay of the Mar. 5, 1979 event.

John Laros, using a solar x-ray detector on ISEE-3, found a third soft repeater (14) in 1985. I then published the 3-SGR source map (15) as evidence for a galactic plane / LMC pattern (Fig. 8). Later repeaters were also found to lie along the galactic disk, enhancing this interpretation. S. Kulkarni (16) found an optically invisible radio snr for one SGR source, prompting T. Murakami to point the ASCA spacecraft at, and detect, an SGR in real time (17). This event had a low-energy x-ray spectrum fitting the transit through the dense galactic plane, confirming the distant galactic pattern and basically resolving this issue. No superflare event 
was found until August, 1998, 19 years after the 1979 March 5 event (18), a bit anticlimactic, since BeppoSAX and J. van Paradijs had just previously ushered in the cosmological GRB era.

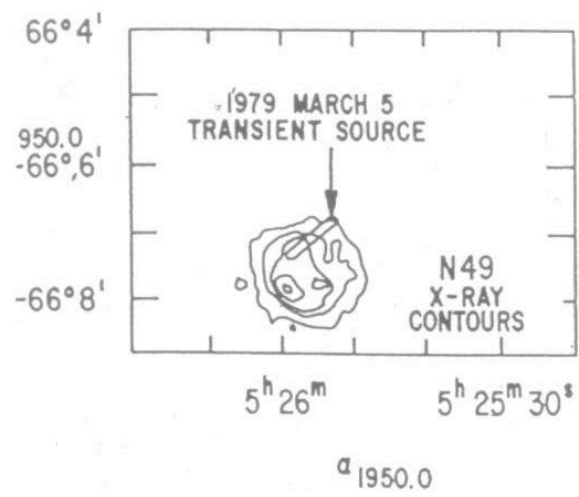

Fig. 7. The reanalyzed N49 source fit.

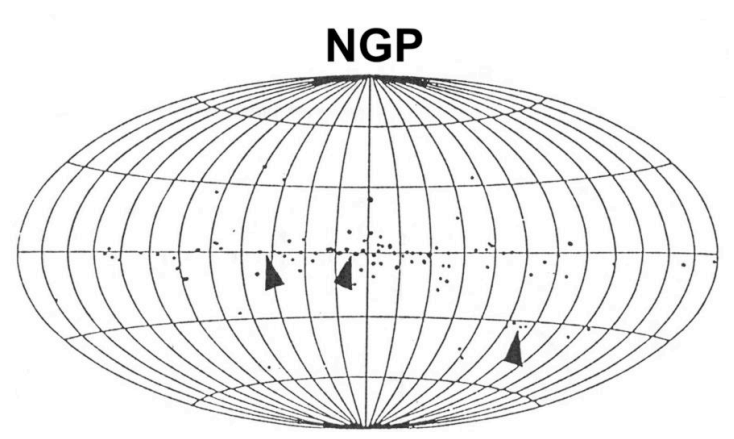

Fig. 8. The first 3 soft repeater sources.

In 1987, I gave an unpublished talk at a meeting dedicated to SN87A, pointing out that instruments on Solar Max did have the LMC in view at the time of the onset of that supernova, but no GRB was found. This was no surprise, of course, since at that time many people assumed that GRBs could not be from such a distant source as the LMC, anyway.

I had previously proposed a large-area, hemispherically sensitive "Gamma-Dome" GRB detector for the first NASA Shuttle. It was competitively accepted, I understand, but all science was later canceled for that mission. When an opportunity came along, later, to propose for the Gamma-Ray Observatory, we at Goddard were fully booked, so I suggested to Jerry Fishman that he propose something along these lines as a service instrument for GRO. As you all know, the BATSE succeeded beyond all expectations, and Jerry always graciously credited my ideas.

B. Schaefer's controversial claim of an archived 1928 optical event in a precise 1978 GRB source location (19) - later reinterpreted as a photographic plate defect (20) - confused the GRB mystery, implying that GRBs repeat, and might be detected easily by optical means. I had unsuccessfully tried to get the Fly's Eye array of Cerenkov sensors modified to look for GRBs, but G. Ricker of MIT built a sea-level optical array, the ETC. This failed to detect GRB optical transients; nevertheless it prompted MIT's High Energy Transient Explorer as the first space mission devoted entirely to GRBs. The first HETE launch failed, giving MIT the opportunity to replace the optical system with an X-ray detector and enjoy an equatorial orbit for HETE-2 (21).

Another opportunity for GRB study was the Solar Polar mission, a pair of spacecraft to orbit the Sun well out of the ecliptic plane. My proposal for twin GRB (and solar flare) detectors, with Kevin Hurley of Toulouse and Michael Sommer of MPE Garching as my coPrincipal Investigators on the companion ESA spacecraft, was accepted. Michael eventually retired, and Kevin brought his project to California. After our instrument was built, the NASA spacecraft was canceled, leaving the renamed Ulysses as a single, 5-AU, IPN vertex. From the mid-80s to early 90s, IPNs had been relatively unsuccessful, with the failure of several Soviet and NASA Mars missions. Also, the initial Ulysses trajectory was in the same direction as Venus, collapsing that triangle just prior to PVO stopping operation, Thus, few high-precision GRB sources were possible until NEAR was finally put into solar orbit for an asteroid 
rendezvous. This IPN, even without BeppoSax involvement, enabled many afterglow prompts, including the most distant source found until that time, at $z=4.5$ (22). One might imagine the success of the IPN in generating afterglow opportunities had Solar-Polar not been eliminated.

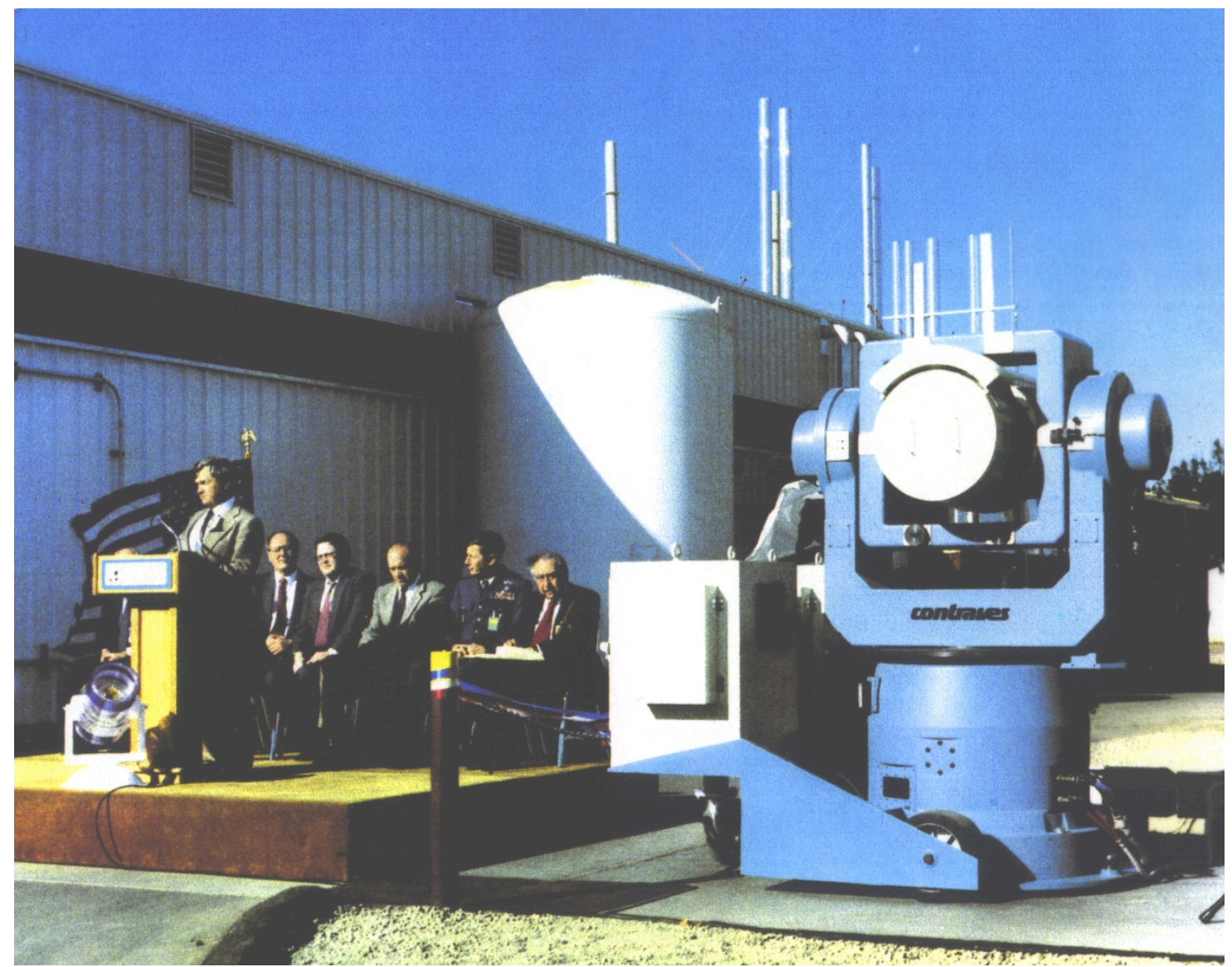

Fig. 9. The first rapid-response GRB afterglow telescope, contributed by the SDI.

Scott Barthelmy's BACODINE system, using GRO-BATSE data directly, and his GCN system, revolutionized GRB research by enabling rapid afterglow studies and inspiring the development of a number of reorienting sea-level telescopes. The first of these, at Livermore (23), was donated from E. Teller's Strategic Defense Initiative. It was originally a 'Star Wars' device built to orbit the Earth for tracking incoming enemy missiles (Fig. 9). Teller, in fact, had given a GRB conference talk years earlier, arguing for the cosmological sources of GRBs. Unfortunately, this was never published. Soon after GRO was launched in 1991, B. Paczinski championed the cosmological model, years before the discovery of its validity with BeppoSax. Second-generation rapid response telescopes were soon developed, and C. Akerlof at Michigan found the first optical afterglow coincident in time with a GRB gamma-ray profile (24) in 1991.

In retrospect, since NASA was formed to compete with the Soviets, it is a curious but fortunate irony that the first Soviet scientific experiment on a NASA spacecraft contrasts the weapons monitoring history and the SDI: the Konus GRB detector. Launched on the GGSWind in 1994 with Goddard interface instrumentation, Konus, reviewed here by R.Aptekar (25), is still working perfectly. My involvement with Konus//Wind has been a real privilege (Fig 10). 


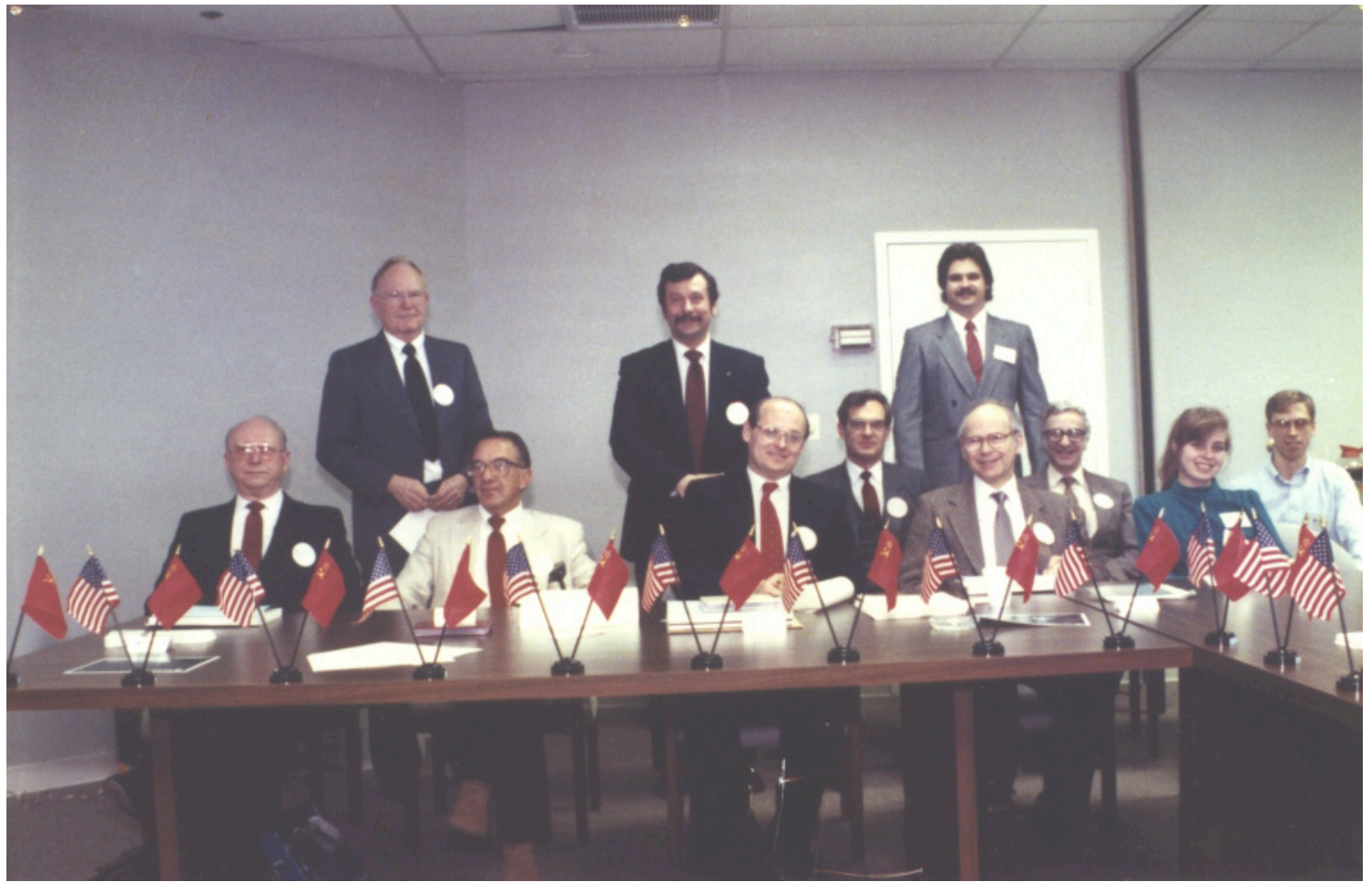

Fig. 10. Celebrating the successful Soviet/American Konus/Wind project.

\section{Acknowledgments}

U. Desai and G. Pizzichini were significant colleagues in the early gamma ray burst era. G. Porreca's craftsmanship made possible the OGO, IMP-6 and -7 instruments, the Helios-2, balloon-borne, ISEE-1 and -3 and Solar Polar GRB detectors, and the Konus/Wind interface subsystem. I also wish to thank P. Tyler for software assistance on many occasions, and to thank the Conference organizers and especially J. Greiner for all the hospitality and generous support.

\section{References}

[1] S. Colgate, Prompt gamma rays and X-rays from supernovae, Can. J. Physics, 46,(1968) 476.

[2] R. Klebesadel, I. Strong, I., and R. Olson, Observations of Gamma Ray Bursts of Cosmic Origin, ApJL, 182 (1973) L85.

[3] T. Cline and U. Desai, Search for Brief Celestial X-ray Bursts, in Proc. 13th ICRC, 80 (1973).

[4] T. L. Cline et al., Energy Spectra of Cosmic Gamma Ray Bursts, ApJL, 185 (1973) L1.

[5] W. A. Wheaton et al., The Direction and Spectral Variability of a Cosmic Gamma Ray Burst, ApJL, 185 (1973) L57.

[6] T. L. Cline et al., No new limit on the size distribution of gamma ray bursts, Nature, 266 (1977) 749.

[7] T. L. Cline et al., Detection of a fast, intense and unusual gamma ray transient, ApJL, 237 (1980) L1. 
[8] W. D. Evans et al., Location of the gamma-ray transient event of 1979 March 5, ApJL, 237 (1980) L7.

[9] E. P. Mazets et al., Observations of a Flaring X-ray pulsar in Dorado, Nature, 282 (1979) 587.

[10] T. L. Cline et al., Precise source location of the anomalous 1979 March 5 gamma-ray transient, ApJL, 255 (1982) L45.

[11] S.V. Golenetskii, V.N. Il'Insky, and E.P. Mazets, Recurrent bursts in GBS0526-66, the source of the March 51979 gamma ray burst, Nature, 307 (1984) 41.

[12] T. L. Cline, The unique cosmic event of 1979 March 5, Comments on Astrophysics, 9 (1980) 13.

[13] J. P. Norris et al., Frequency of fast, narrow gamma-ray bursts, Nature, 308 (1984) 434.

[14] J. Laros et al., A new type of repetitive behavior in a high-energy transient, ApJL, 320 (1987) 1111.

[15] T. L. Cline, Gamma Ray Transients, in Essays in Space Science, NASA CP-2464 (1985) 295.

[16] S. Kulkarni and D. Frail, Identification of a supernova remnant coincident with the soft gammaray repeater SGR1806-20, Nature, 365 (1993) 127.

[17] T. Murakami et al., X-ray identification of the soft $\gamma$-ray repeater 1806-20, Nature, 368 (1994) 127.

[18] K. Hurley et al., A giant periodic flare from the soft $\gamma$-ray repeater SGR1900+14, Nature, 397 (1999) 41.

[19] B. E. Schaefer, Probable optical counterpart of a Gamma-ray burster, Nature, 294 (1981) 722.

[20] J. Greiner, Rejection of archival photographic objects as optical counterparts of gamma-ray bursters, A \& A, 264 (1992) 121.

[21] G. Ricker et al., The High Energy Transient Explorer (HETE): Mission and Science Overview, AIP Conf. Proc., 662 (2003) 3.

[22] M. Andersen et al., VLT Identification of the optical afterglow of the gamma ray burst GRB000131 at $z=4.50$, Astron. Astrophys., 364 (2000) L54.

[23] H-S. Park et al., Realtime Tracking system for the wide-field-of-view telescope project, SPIE Proc., 1111 (1989) 196.

[24]C. Akerlof et al., Observation of contemporaneous optical radiation from a $\gamma$-ray burst, Nature, 398 (1999) 400.

[25] R. Aptekar, "Cosmic gamma ray burst studies with Ioffe Institute Experiments" in Proceedings of the "Gamma Ray Bursts 2012 Conference", PoS(GRB2012)118 